\title{
Haemodynamic effects of increasing angle of head up tilt
}

\author{
A Zaidi, D Benitez, P A Gaydecki, A Vohra, A P Fitzpatrick
}

\begin{abstract}
Objective-To investigate the haemodynamic effects of varying the angle of head up tilt. Methods-20 healthy subjects (12 female, eight male; mean (SD) age 33.6 (8.4) years) underwent head up tilt for five minutes to each of four angles of tilt in random order, with a five minute rest period at the horizontal between each angle. Forearm blood flow was measured using intermittent occlusion mercury strain gauge plethysmography at two and five minutes. Subjects underwent continuous monitoring of heart rate, systolic blood pressure (SBP), and diastolic blood pressure (DBP) by Finapres and cardiac output and stroke volume by impedance cardiography. Each variable was measured at two and five minutes, averaged over the period of blood flow measurement.

Results-Every haemodynamic variable at each angle was significantly different from supine values. Head up tilt produced progressive increases in heart rate (11-21\%), SBP (12-21\%), and DBP $(20-33 \%)$ with increasing tilt angle. However, although $45^{\circ}$ produced significantly less haemodynamic effect, there were no significant differences for angles between $60^{\circ}$ and $90^{\circ}$. Cardiac output fell on head up tilt by $17-20 \%$ and stroke volume by $28-34 \%$, but increasing tilt angle produced no significant additional reduction in cardiac output and stroke volume because of increases in heart rate and vasoconstriction.

Conclusions-Angles $<60^{\circ}$ produce significantly less haemodynamic effects than steeper angles. Increasing tilt angle beyond $60^{\circ}$ produces no apparent additional effect on cardiac output or sympathetic tone. Increasing tilt angle beyond $60^{\circ}$ confers no additional orthostatic stress and may not affect the sensitivity and specificity of head up tilt testing as previously thought. Sixty degrees of tilt is a more practical angle for support of a syncopal patient and is recommended. (Heart 2000;83:181-184)
\end{abstract}

Keywords: head up tilt test; vasovagal syncope; thoracic impedance

Head up tilt testing is established as the key investigation in the diagnosis of vasovagal syncope. However, despite the widespread use of tilt testing, there is no agreement on a standard protocol. Various angles and durations of tilt are used, with or without provocative agents, including isoprenaline, ${ }^{1-4}$ edrophonium, ${ }^{5}$ and glyceryl trinitrate. ${ }^{6}$ Most early tilt studies used passive $60^{\circ}$ tilt but this required up to $60 \mathrm{~min}$ utes of head up tilt to achieve satisfactory sensitivity. ${ }^{7-10}$ Steeper angles of tilt have been used to reduce the duration of the test, ${ }^{1-4}{ }^{11}$ and the perception has grown that steeper levels of tilt confer additional orthostatic stress, increasing peripheral venous pooling and $\beta$ adrenergic stimulation, ${ }^{12}$ thereby increasing the sensitivity of tilt testing at the expense of reduced specificity. However, although there have been studies comparing the effects of different tilt angles using the development of syncope or presyncope as the end point, ${ }^{413}$ the haemodynamic effects of increasing the angle of tilt remain unclear. In this study we investigated the haemodynamic effects of varying tilt angle to determine the most appropriate angle for head up tilt testing.

\section{Methods}

SUBJECT POPULATION

We studied 20 healthy controls (12 female, eight male; mean (SD) age 33.6 (8.4) years) with no history of syncope or presyncope. All subjects were hospital medical personnel.
None of the subjects had a history of cardiovascular disease or was taking any cardioactive drugs. Informed consent was obtained in each case.

TILT TESTING PROTOCOL

Tilt testing took place in quiet, dimly lit room with subjects in a postabsorptive state. Subjects were comfortably restrained on an electric tilt table with foot plate support. Surface electrocardiography (Hewlett-Packard 78351A ECG monitor, Hewlett-Packard Co, Andover, Massachusetts, USA) was used for continuous monitoring of heart rate and rhythm. Beat to beat blood pressure was monitored continuously with a non-invasive finger tip photoplethysmographic device (Finapres 2300 BP monitor, Ohmeda, Englewood, Colorado, USA). Thoracic impedance was continuously monitored using the BoMed NCCOM3-R7S impedance cardiograph (BoMed Medical Manufacturing Co, Irvine, California, USA), using the technique for non-invasive measurement of cardiac output and stroke volume previously described by Kubicek et al. ${ }^{14}$ Forearm blood flow was measured by intermittent cuff occlusion mercury-in-Silastic strain gauge plethysmography ${ }^{15}$ (EC4 plethysmograph, DE Hokanson Inc, Bellevue, Washington, USA).

Analogue signals from each recorder were collected through BNC connectors by a 16 bit data acquisition card (National Instruments PCI-MIO-16XE-50) plugged into the PCI bus 
Table 1 Cardiovascular response to varying angle of head up tilt

\begin{tabular}{|c|c|c|c|c|c|c|c|c|c|}
\hline \multirow{2}{*}{$\begin{array}{l}\text { Tilt angle } \\
\text { Rest }\end{array}$} & \multirow{2}{*}{$\frac{H R \text { (beats } / \mathrm{min})}{62.2(8.1)}$} & \multirow{2}{*}{$\begin{array}{l}S B P(m m ~ H g) \\
122.4(22.6)\end{array}$} & \multirow{2}{*}{$\begin{array}{l}\mathrm{DBP}(\mathrm{mm} \mathrm{Hg}) \\
69.4(12.9)\end{array}$} & \multicolumn{2}{|c|}{$\mathrm{CO}(\mathrm{l} / \mathrm{min})$} & \multicolumn{2}{|c|}{$S V(m l)$} & \multicolumn{2}{|c|}{$F B F(\% / \mathrm{min})$} \\
\hline & & & & 6.4 & $(1.9)$ & 104.8 & $(34.4)$ & 2.43 & $(1.34)$ \\
\hline $45^{\circ}$ & $69.1(11.3)$ & $137 \quad(23.2)$ & $83.5(15.7)$ & 5.3 & (1.6) & 75.8 & $(20.7)$ & 1.96 & $(1.22)$ \\
\hline $60^{\circ}$ & $72.7(11.3)$ & $138.7(19.5)$ & $88.1(13.5)$ & 5.3 & (1.6) & 72.6 & (21.4) & 1.55 & $(0.81)$ \\
\hline $75^{\circ}$ & $73.9(10.6)$ & $141.3(26.2)$ & $88.2(17.3)$ & 5.1 & (1.6) & 68.9 & (20.6) & 1.56 & $(0.74)$ \\
\hline $90^{\circ}$ & $75.2(11.0)$ & $148.6(25.5)$ & $92 \quad(17.1)$ & 5.2 & (1.7) & 69.5 & $(21.9)$ & 1.31 & $(0.86)$ \\
\hline $\mathrm{F}(4,60)$ & 34.5 & 21.8 & 50.7 & 12.1 & & 35.9 & & 8.8 & \\
\hline $\mathrm{p}$ Value & $<0.001$ & $<0.001$ & $<0.001$ & 0.00 & & $<0.00$ & & 0.001 & \\
\hline
\end{tabular}

*Comparison between supine and different angles of tilt made separately for each haemodynamic variable using single factor repeated measures analysis of variance.

$\mathrm{CO}$, cardiac output; DBP, diastolic blood pressure; FBF, forearm blood flow; HR, heart rate; SBP, systolic blood pressure; SV, stroke volume.

of a pentium PC using LabVIEW(R) software (National Instruments Corporation, Austin, Texas, USA). Analogue data were sampled simultaneously at $1000 \mathrm{~Hz}$ and saved directly to the hard disk for later off-line analysis using purpose designed algorithms.

Following the positioning of the non-invasive recorders, subjects were allowed to rest in the supine position for 20 minutes. After this period of equilibration and completion of baseline haemodynamic measurements, subjects underwent passive head up tilt to each of four angles of tilt in random order. On the basis of previous studies showing that haemodynamic steady state was reached within five minutes of the onset of tilt in healthy controls, ${ }^{1617}$ subjects were tilted for five minutes, with a five minute rest period at the horizontal between each angle. Haemodynamic measurements were made two and five minutes after reaching the angle under study, averaged over the 15 second period of forearm blood flow measurement.

DATA ANALYSIS

Data were analysed for each variable separately using single factor repeated measures analysis of variance. No transformations were applied to the data. Tukey's HSD test was used to identify differences between different angles of tilt for each haemodynamic variable. A $\mathrm{p}$ value $<0.05$ was considered significant.

\section{Results}

No patients developed symptoms during head up tilt. Data were not significantly different at two and five minutes of tilt. Subsequent results relate to data recorded at five minutes of head up tilt and are summarised in tables 1 and 2 . Data are expressed as mean (standard deviation). All haemodynamic variables were significantly different at each angle of tilt compared with supine (table 1). Head up tilt produced progressive increases in heart rate $(11-21 \%)$, systolic blood pressure (12-21\%), and diastolic

Table 2 Change in haemodynamics from supine to each angle of tilt

\begin{tabular}{lllllll}
\hline Tilt angle & $\% H R$ & $\% S B P$ & $\% D B P$ & $\% C O$ & $\% S V$ & $\% F B F$ \\
\hline $45^{\circ}$ & 11.1 & 11.9 & 20.3 & -17.2 & -27.7 & -19.3 \\
$60^{\circ}$ & $16.9^{\star}$ & 13.3 & 26.9 & -17.2 & -30.7 & $-36.2^{\star}$ \\
$75^{\circ}$ & $18.8^{\star}$ & 15.4 & 27.1 & -20.3 & -34.3 & $-35.8^{\star}$ \\
$90^{\circ}$ & $20.9^{\star}$ & $21.4^{\star}+$ & $32.6^{\star}$ & -18.8 & -33.7 & $-46.1^{\star}$
\end{tabular}

$\star$ Significant difference from $45^{\circ}$; † significant difference from $60^{\circ}(\mathrm{p}<0.05)$.

$\% \mathrm{CO}$, percentage change in cardiac output after tilting; \% DBP, percentage change in diastolic blood pressure after tilting; \% FBF, percentage change in forearm blood flow after tilting; \% $\mathrm{HR}$, percentage change in heart rate after tilting; \% SBP, percentage change in systolic blood pressure after tilting; \% SV, percentage change in stroke volume after tilting. blood pressure (20-33\%) with increasing tilt angle. Cardiac output fell on head up tilt by $17-20 \%$ and stroke volume by $28-34 \%$, but increasing tilt angle produced no significant additional reduction in cardiac output and stroke volume because of increases in heart rate and vasoconstriction, indicated by progressive falls in forearm blood flow. Tilting to $45^{\circ}$ produced less relative tachycardia and vasoconstriction than tilting to greater angles and a significantly smaller increase in systolic and diastolic blood pressure from supine than at $90^{\circ}$. The only significant difference between angles between $60^{\circ}$ and $90^{\circ}$ was in systolic blood pressure, which was higher at $90^{\circ}$ than at $60^{\circ}$ (table 2).

\section{Discussion}

HAEMODYNAMIC EFFECTS AT ANGLES BELOW $60^{\circ}$ The ACC expert consensus document on tilt table testing for assessing syncope concluded that angles below $60^{\circ}$ provide insufficient orthostatic stress and result in a lower yield of positives in patients with syncope. ${ }^{18}$ There is compelling evidence to support this view. Haemodynamic studies in healthy controls have shown no significant haemodynamic effect at angles below $30^{\circ} .{ }^{19}$ At angles between $30^{\circ}$ and $60^{\circ}$ there was a linear response in cardiac output, diastolic blood pressure, and heart rate, with no significant change in systolic blood pressure, ${ }^{16}{ }^{19}$ while at angles beyond $60^{\circ}$ there was no significant change in cardiac output. ${ }^{19}$ This is reflected in the apparent loss of sensitivity in tilt testing at angles below $60^{\circ}$. Fitzpatrick et al reported that passive tilting to $45^{\circ}$ reproduced syncope in only three of 10 patients with positive tests at $60^{\circ},{ }^{20}$ while Sander-Jensen et al showed that $30^{\circ}$ tilt failed to reproduce syncope in healthy controls with typical vasovagal reactions at $60^{\circ}$ using a saddle support. ${ }^{21}$

CLINICALLY USEFUL TILT ANGLES

For angles between $60^{\circ}$ and $80^{\circ}$, the conclusion of the ACC expert consensus document was that there was no substantial difference in terms of test outcome in the absence of pharmacological provocation, but that there was a higher positive yield at steeper tilt angles with isoprenaline provocation. ${ }^{18}$ This implies that tilt angles beyond $60^{\circ}$ confer additional orthostatic stress, but the evidence for this is circumstantial. In general, series of head up tilt testing have shown a higher yield with $80^{\circ}$ tilt than with $60^{\circ}$ tilt. $^{1-4}{ }^{7-11}$ However, positivity 
rates of $75 \%$ have been achieved using passive $60^{\circ}$ tilt for 60 minutes, ${ }^{20}$ which compares favourably with most studies using $80^{\circ}$, and it seems probable that patient selection rather than additional orthostatic stress accounts for much of the apparent superiority of $80^{\circ}$ tilt. Furthermore, the majority of series at $80^{\circ}$ have used provocative agents, which clearly increase the positive yield of tilt testing. ${ }^{1-411}$ There are no satisfactory studies comparing the haemodynamic effects of head up tilting to $60^{\circ} v 80^{\circ}$. However, Voice et al compared the susceptibility to syncope at the two most widely used angles for clinical tilt testing by randomising patients with two or more episodes of unexplained syncope to 45 minute passive tilt at $60^{\circ}$ or $80^{\circ} .{ }^{4}$ The positive yield was higher at $80^{\circ}$ ( $54 \%$ compared with $42 \%$ at $60^{\circ}$ ), but neither the positive rate nor the time to syncope were significantly different and it was concluded that there was probably little difference between the two angles.

\section{ISOPRENALINE STUDIES}

The best evidence for the superiority of $80^{\circ}$ comes from studies of head up tilt in healthy controls, particularly with isoprenaline provocation. Natale et al randomised healthy controls to tilt at $60^{\circ}, 70^{\circ}$, or $80^{\circ}$ (20 minutes passive tilt followed by tilt with isoprenaline infusion to raise heart rate by $20 \%) .{ }^{13}$ During baseline tilt, there was reduced specificity with increased tilt angle (positive rate $8 \%$ at $60^{\circ}, 8 \%$ at $70^{\circ}, 20 \%$ at $80^{\circ}$ ) but the difference in the positive rate between the different tilt angles was not significant. Isoprenaline infusion produced similar results (positive rate $4 \%$ at $60^{\circ}, 4 \%$ at $70^{\circ}, 28 \%$ at $80^{\circ}$ ), with significant differences between $80^{\circ}$ and the two lower angles, albeit with small numbers of false positives. However, the specificity of $80^{\circ}$ tilt with isoprenaline has been shown to be as high as $92 \%$ in other studies. ${ }^{22}$ Similarly, Kapoor et al pooled the results of 14 tilt test series using isoprenaline at various angles between $60^{\circ}$ and $90^{\circ}$ and showed that the proportion of positive tests rose progressively with increasing tilt angle, suggesting that orthostatic stress increases with tilt angle. ${ }^{23}$ However, the data for $70^{\circ}$ and $90^{\circ}$ were from single small studies and the lack of a uniform protocol for patient selection and investigation raises concern about patient selection bias.

PATHOPHYSIOLOGY OF HEAD UP TILT

The pathophysiology of tilt induced syncope remains incompletely understood but it is thought that the primary effect of orthostasis is the redistribution of up to $1000 \mathrm{ml}$ of blood from the central cardiovascular compartment to the capacitance vessels of the lower limbs. ${ }^{24}$ This leads to a progressive fall in venous return, which may be excessive in patients predisposed to vasovagal syncope ${ }^{25}$ resulting in inappropriate activation of left ventricular mechanoreceptors ${ }^{26}$ and paradoxical reflex sympathetic withdrawal. ${ }^{27}$ It has been assumed that by increasing the tilt angle the degree of gravity mediated displacement of the circulating volume to the peripheries is also increased. However, the redistribution of blood is directly related to the sine of the angle of tilt ${ }^{28}$ which rises in an approximately linear fashion between $30^{\circ}$ and $60^{\circ}$ but changes very little at angles beyond $60^{\circ} \quad\left(\sin 60^{\circ}=0.87, \sin \right.$ $80^{\circ}=0.98$ ). This is in keeping with previous haemodynamic studies at angles below $60^{\circ 16} 19$ and with our present results, and suggests that there is a "plateauing" of haemodynamic effects at $60^{\circ}$, rendering higher angles of tilt unnecessary. The haemodynamic change seen in our control subjects is similar to previous studies using passive $90^{\circ}$ tilt in controls, where stroke volume fell by approximately $28 \%$ on tilt with a $30 \%$ increase in peripheral vascular resistance. $^{29}$

\section{CONCLUSIONS}

Sixty degrees of tilt is the most practical angle for supporting syncopal patients. Increasing tilt angle beyond $60^{\circ}$ produces no additional orthostatic stress. This suggests that passive tilt at $60^{\circ}$ for up to 60 minutes is the optimum protocol for achieving the combination of high specificity with low sensitivity in head up tilt testing.

1 Almquist A, Goldenberg IF, Milstein S, et al. Provocation of bradycardia and hypotension by isoproterenol and upright posture in patients with unexplained syncope. N Engl F Med 1989;320:346-51.

2 Kapoor WN, Brant N. Evaluation of syncope by upright tilt testing with isoproterenol. Ann Intern Med 1992;116:35862.

3 Sheldon R, Killam S. Methodology of isoproterenol-tilt able testing in patients with syncope. $\mathcal{F}$ Am Coll Cardiol 1992;19:773-9.

4 Voice RA, Lurie KG, Sakaguchi S, et al. Comparison of tilt angles and provocative agents (edrophonium and isoproterenol) to improve head-upright tilt-table testing. Am $\mathcal{f}$ Cardiol 1998;81:346-50.

5 Lurie KG, Dutton J, Mangat R, et al. Evaluation of drophonium as a provocative agent for vasovagal syncope in patients with syncope. Am f Cardiol 1993;72:1288-90.

6 Raviele A, Gasparini G, Di Pede, et al. Nitroglycerin infusion during upright tilt: a new test for the diagnosis of vasovagal syncope. Am Heart $\mathcal{7}$ 1994;127:103-11.

7 Fitzpatrick AP, Sutton R. Tilting towards a diagnosis in recurrent unexplained syncope. Lancet 1989;i:658-60.

8 Raviele A, Gasparini G, Di Pede, et al. Usefulness of head-up tilt test in evaluating patients with syncope of head-up tilt test in evaluating patients with syncope of
unknown origin and negative electrophysiologic study. $\mathrm{Am}$ unknown origin and negatiol $1990 ; 65: 1322-7$.

9 Strasberg B, Rechavia E, Sagie A, et al. The head-up tilt table test in patients with syncope of unknown origin. $A m$ Heart f 1989;118:923-7.

10 Abi-Samra F, Maloney JD, Fouad-Tarazi FM, et al. The usefulness of head-up tilt testing and hemodynamic investigations in the workup of syncope of unknown origin. PACE 1988;11:1202-14

11 Grubb B, Temesy-Armos P, Hahn H, et al. Utility of upright tilt-table testing in the evaluation and management of syncope of unknown origin. Am f Med 1991;114:1013-19.

12 Mark AL. The Bezold-Jarisch reflex revisited: clinical implications of inhibitory reflexes originating in the heart. $\mathcal{F} \mathrm{Am}$ Coll Cardiol 1983;1:90-102.

13 Natale A, Akhtar M, Jayazeri M, et al. Provocation of hypotension during head-up tilt testing in subjects with no history of syncope or presyncope. Circulation 1995;92:54-8.

14 Kubicek WG, Patterson RP, Wilson DA. Impedance cardiography as a non-invasive method of monitoring carcardiography as a non-invasive method of monitoring car-
diac function and other parameters of the cardiovascular system. Ann NY Acad Sci 1970;170:724-32.

15 Hokanson DE, Sumner DS, Strandness DE. An electrically calibrated plethysmograph for direct measurement of limb blood flow. IEEE Trans Biomed Eng 1975;22:21-5.

16 Hainsworth R, Al-Shamma YMH. Cardiovascular responses to upright tilting in healthy subjects. Clin Sci 1988; $74: 17-22$.

17 Dikshit MB, Banerjee PK, Rao PLNB. Orthostatic tolerance of normal Indians and those with suspected abnormal cardiovascular reflexes. Aviat Space Envir Med 1986;57: 168-73.

18 Benditt DG, Ferguson DW, Grubb BP, et al. Tilt table testing for assessing syncope. 7 Am Coll Cardiol 1996;28:263-

19 Matalon SV, Fahri LE. Cardiopulmonary readjustments in passive tilt. F Appl Physiol 1979;47:503-7.

20 Fitzpatrick AP, Theodorakis G, Vardas P, et al. Methodology of head-up tilt testing in patients with unexplained of head-up tilt testing in patients with
syncope. $\mathcal{F} \mathrm{Am}$ Coll Cardiol 1991;17:125-30.

21 Sander-Jensen K, Secher NH, Astrup A, et al. Hypotension induced by passive head-up tilt: endocrine and circulatory mechanisms. Am f Physiol 1986;251:R743-9. 
22 Grubb BP, Kosinski D, Temesy-Armos P, Brewster responses of normal subjects during $80^{\circ}$ head upright tilt
testing with and without low dose isoproterenol infusion. testing with and without

23 Kapoor WN, Smith MA, Miller NL. Upright tilt testing in evaluating syncope: a comprehensive literature review. $\mathrm{Am}$ $7 \mathrm{Med} 1994 ; 97: 78-88$

24 McMichael J, Sharpey-Schafer EP. Cardiac output in man by a direct Fick technique; effects of posture, venous pressure change, atropine and adrenalin. Br Heart 7 1944; 6:3340 .
25 Rea R, Thomas M. Neural control mechanisms and vasovagal syncope. F Cardiovasc Electrophysiol 1993;4:587-95.

( control. Rev Physiol Biochem Pharmacol 1979;86:1-94.

27 Wallin BG, Sundlof G. Sympathetic outflow in muscles during vasovagal syncope. $\mathscr{F}$ Auton Nerv Syst 1982;6:287-91. 28 Hellebrandt FA, Franseen EB. Physiological study of the vertical posture in man. Physiol Rev 1943:23:220-5.

29 Stevens PM. Cardiac dynamics during orthostasis and the influence of intravascular instrumentation. Am 7 Cardiol 1966;17:211-18.

\section{IMAGES IN CARDIOLOGY}

\section{Aortic valve tumour trapped in the right coronary ostium}
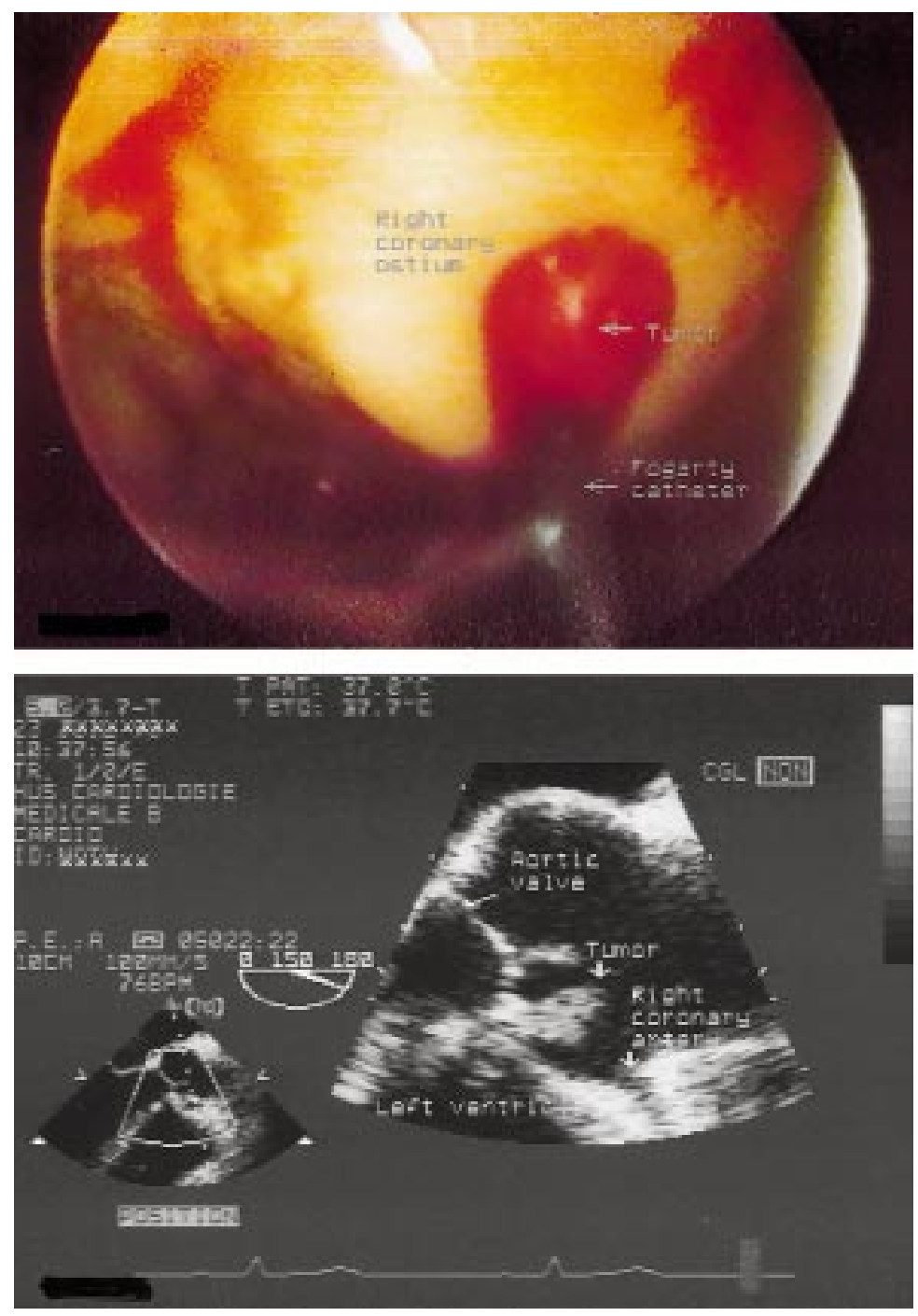

A 48 year old man with a history of rectocolitis was admitted to a coronary care unit because of an inferior myocardial infarction. Revascularisation was not performed because of the absence of pain at the time of admission and delay (six hours) after the onset of thoracic pain. The patient had no risk factors for atherosclerosis apart from smoking, which he had stopped two years ago.

Transthoracic cross sectional echocardiography revealed an abnormal structure behind the right coronary aortic cusp. This was confirmed by multiplane transoesophageal echocardiography, which showed a pedunculated tumour overhanging the right coronary ostium. Because of the risk of embolisation, coronary angiography was not performed and surgical removal of the tumour was attempted. At surgery, behind the right coronary cusp, only remnants of the tumour were found and excised. Peroperative videoscopy detected the tumour trapped in the right coronary ostium. The tumour (7 $\mathrm{mm}$ diameter) was extracted with a Fogarty catheter. Histopathology showed a fibroma with areas of attached thrombus. One month after surgery, coronary angiography was performed, which showed single vessel disease with a $70 \%$ distal stenosis of the right coronary artery. Because of inferior akinesia of the left ventricle and negative stress test, coronary revascularisation was not attempted.

PIERRE ATTALI PHILIPPE BILLAUD JEAN-MARIE MOSSARD 